\title{
Expression of CD15 in normal and metaplastic Paneth cells of the digestive tract
}

\author{
A Ariza, D López, E M Castellà, C Muñoz, M J Zújar, J L Mate
}

\begin{abstract}
Aims-To substantiate that incubation with monoclonal antibody CD15 (C3D-1) elicits a distinctive immunoreaction in normal small intestinal Paneth cells, normal and metaplastic Paneth cells along the digestive tract were assessed to determine whether they are also immunoreactive to CD15.

Methods-Paneth cells in paraffin wax embedded specimens of normal small intestine, appendix and proximal ascending colon, and from cases of chronic gastritis and ulcerative colitis were investigated immunohistochemically for lysozyme and CD15 antigen expression by means of the avidin-biotin peroxidase complex method.

Results-CD15 antibody reacted with a high proportion of both normal and metaplastic Paneth cells. Paneth cell immunoreactivity to CD15, however, was less intense and less extensive than to antilysozyme antibody, though the latter also stained many other cell types and was more commonly associated with nonspecific background staining.

Conclusions-CD15 seems to be a valuable adjuvant for the study of Paneth cells in the normal and diseased digestive tract. Furthermore, as CD15 has been shown to be involved in activation of phagocytes, its expression in Paneth cells reinforces their proposed role as antimicrobial agents and regulators of the intestinal flora.

( Clin Pathol 1996;49:474-477)
\end{abstract}

Keywords: CD15, immunohistochemistry, lysozyme, Paneth cell.

Paneth cells constitute one of the four major epithelial cell types of the mucosa of the small intestine, caecum, appendix, and proximal ascending colon..$^{1-3}$ They also display a well known tendency to appear in many other anatomical locations in a variety of pathological conditions. Thus, Paneth cells are often present in other segments of the digestive tract, such as the stomach in chronic gastritis and colon in ulcerative colitis. ${ }^{45}$ Moreover, elements variably construed as Paneth or Panethlike cells have been detected outside the digestive tract-for example, in the testis, ${ }^{6}$ the uterine cervix, ${ }^{7}$ and the prostate. ${ }^{89}$

In spite of the highly characteristic histological appearance displayed by Paneth cells (pyramidal shape and supranuclear, intensively eosinophilic granules), other cell types can mimic them quite effectively. ${ }^{6-9}$ Discrimination between Paneth-like changes and true Paneth cells is best achieved by means of immunohistochemistry, but this approach is hampered by a paucity of adequate markers. Lysozyme content, ${ }^{3}$ shared with many other cell types, is virtually the only feature that can be used by pathologists to achieve immunohistochemical recognition of Paneth cells. Investigation of other markers, such as a zinc binding protein (ZBPP-1) ${ }^{10}$ is not readily available or feasible in most histopathology laboratories, and the same can be said for pokeweed lectin binding, ${ }^{11}$ group II phospholipase A2, ${ }^{12}{ }^{13}$ tumour necrosis factor $\alpha,{ }^{14}{ }^{15} \mathrm{CD} 1 \mathrm{mRNA},{ }^{16}$ or anionic sites. ${ }^{17}$

We have observed that incubation with monoclonal antibody CD15 gives rise to a positive immunoreaction in normal small intestinal Paneth cells. To confirm this finding, we have addressed the question of whether normal and metaplastic Paneth cells in various locations in the digestive tract are immunoreactive to CD15. Confirmation of CD15 as a marker for Paneth cells may improve our ability to detect these cells immunohistochemically and to elucidate their function.

\section{Methods}

Five archival cases of right hemicolectomy for carcinoma of the ascending colon were retrieved from the archives of the Department of Pathology, Hospital Universitari Germans Trias i Pujol. Haematoxylin and eosin stained sections were examined for histological evidence of Paneth cells and representative blocks were selected (one each from normal terminal ileum, appendix, and proximal ascending colon) from each case.

Metaplastic Paneth cells are generally present in chronic gastritis and ulcerative colitis. Five examples of each with a large number of metaplastic Paneth cells were retrieved from the archives: gastrectomy (carried out because of carcinoma) specimens for chronic gastritis and colectomy specimens for ulcerative colitis (table 1). One block per case (from the left colon in the cases of ulcerative colitis) was selected. All samples had been fixed in $10 \%$ buffered formalin, processed routinely, and embedded in paraffin wax.

\section{IMMUNOHISTOCHEMISTRY}

Sections, $5 \mu \mathrm{m}$ thick, were cut from each block and deparaffinised. The tissue was hydrated, heated in a microwave oven $(3 \times$ three minutes, 
Table 1 CD15 immunoreactivity in Paneth cells

\begin{tabular}{llll}
\hline Tissue type & No. of cases & Extent $(\%)^{\star}$ & Intensity \\
\hline Normal ileum & 5 & $100,100,50,50,75$ &,,,,++++++++++++ \\
Normal appendix & 5 & $100,75,50,50,100$ &,,,,++++++++++++ \\
Normal ascending colon & 5 & $100,100,50,0,100$ &,,,,+++++++++++ \\
Chronic gastritis & 5 & $75,75,100,100,100$ &,,,,+++++++++++++ \\
Ulcerative colitis & 5 & $50,100,100,100,100$ &,,,,+++++++++++++
\end{tabular}

*Percentage of Paneth cells with CD15 immunoreactivity in each of the five cases for each tissue type.

$\star \star$ Intensity of CD15 immunostaining in each of the five cases for each tissue type; score:

$-=$ absent $+=$ weak; $++=$ moderate; $+++=$ strong.

with two minute intervals) in buffered citrate (citric acid and sodium citrate, $\mathrm{pH} 6.0$ ), and incubated with rabbit serum (30 minutes). Sections were then incubated with CD15 (mouse monoclonal antibody directed against purified neutrophils from normal human peripheral blood (clone C3D-1; Dako, Glostrup, Denmark)) at a dilution of 1 in 50 for 22 hours at room temperature. The slides were washed and incubated with biotinylated rabbit anti-mouse immunoglobulin antibodies (diluted 1 in 700), placed in phosphate buffered saline (PBS) containing $6 \%$ hydrogen peroxide for 15 minutes at room temperature, and incubated with the avidin-biotin peroxidase complex (Dako).

To detect expression of lysozyme, sections were digested with saponin (30 minutes) and incubated with swine serum (30 minutes). Sections were then incubated with rabbit antihuman lysozyme polyclonal antibody (Dako), diluted 1 in 300 , for 22 hours at room temperature. The slides were washed and incubated with biotinylated swine anti-rabbit immunoglobulin at a 1 in 1000 dilution and then with the avidin-biotin peroxidase complex (Dako). 3,3'-diaminobenzidine tetrachloride (Serva, Heidelberg, Germany) was used as the chromogen and the sections were counterstained with Harris's haematoxylin. In the controls a non-immune mouse serum replaced
CD15 and a non-immune rabbit serum was used in lieu of the anti-lysozyme polyclonal antibody.

Evaluation of all stains was carried out simultaneously by the authors on a multihead microscope. The number of cells immunostained was calculated as a percentage $(0,25$, 50 , or $100 \%$ ) of the number of Paneth cells identified as such on the haematoxylin and eosin stained sections. The intensity of immunoreactivity was graded as absent $(-)$, weak $(+)$, moderate $(++)$, or strong $(+++)$.

\section{Results}

On staining with haematoxylin and eosin abundant Paneth cells were observed in the crypts of the normal terminal ileum, appendix, and proximal ascending colon in all five right hemicolectomy specimens. Variable numbers of metaplastic Paneth cells were also observed in the cases of chronic gastritis and ulcerative colitis. When the haematoxylin and eosin and immunohistochemical stains were compared (figs $1 \mathrm{~A}-\mathrm{C}$ ), the anti-lysozyme antibody stained $90-100 \%$ of the Paneth cells identified on the haematoxylin and eosin sections. The proportion detected using the CD15 antibody oscillated between 50 and $100 \%$. In one of the samples of normal colonic mucosa, however, none of the Paneth cells was highlighted by either antibody. There was no difference in staining of normal and metaplastic Paneth cells with CD15.

CD15 expression in Paneth cells was visualised as diffuse, slightly granular cytoplasmic staining, often accompanied by distinct staining of the plasma membrane. The CD15 signal was often less intense (moderate to strong) than that achieved on staining with the anti-lysozyme antibody.

A continuous, well defined linear CD15 signal highlighted the enterocyte brush border in almost every instance. CD15 immunoreactiv-
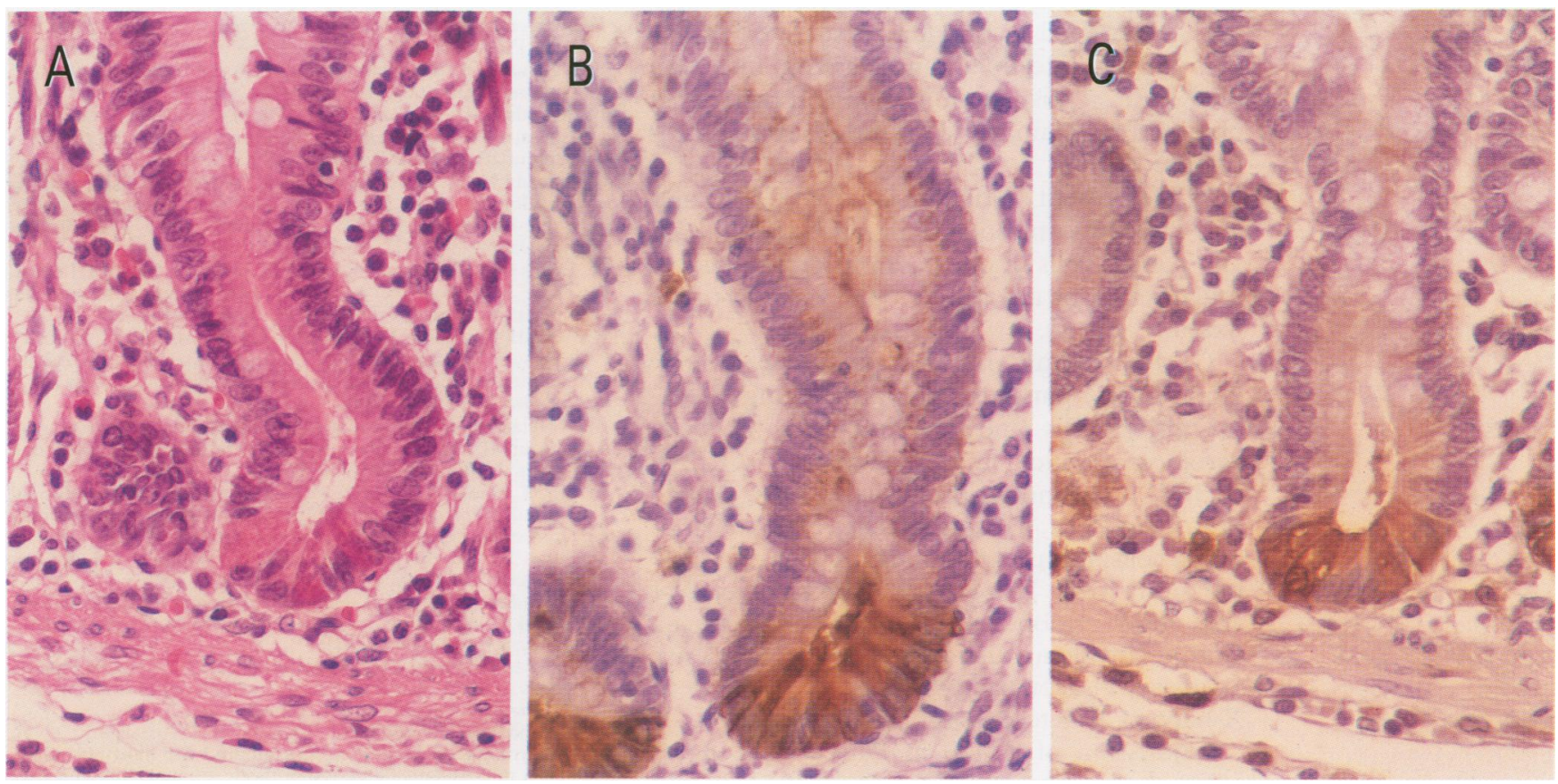

Figure 1 Normal small intestinal Paneth cells on staining with (A) haematoxylin and eosin, and (B) CD15 and (C) anti-lysozyme antibodies ( $\times 400)$. 
ity was observed in goblet cell vacuoles and single, paranuclear dot-like structures, possibly enterocyte Golgi apparatus, were also seen. However, Paneth cells could be readily distinguished by their pancytoplasmic positivity and triangle shaped outline.

CD15 positive granulocytes in the lamina propria were also observed. The anti-lysozyme antibody, however, usually stained much greater numbers of inflammatory cells and resulted in a higher degree of non-specific background staining. Table 1 summarises these results.

\section{Discussion}

Of the four major epithelial cell types of the small intestinal mucosa (absorptive, goblet, endocrine, and Paneth), the one that has remained the most enigmatic is the Paneth cell. ${ }^{12}$ Although more than a century has elapsed since this cell first came to the attention of Josef Paneth, its exact function is still unkown. Recently, however, the identification of Paneth cells as producers of antimicrobial polypeptides (defensins or cryptdins), suggesting that these cells may play a crucial role as regulators of the intestinal microbial flora, has elicited renewed interest in their biology. ${ }^{1819}$

As illustrated by Adlakha and Bostwick, ${ }^{9}$ and others, ${ }^{8}{ }^{20}{ }^{21}$ lysozyme has been the only Paneth cell marker of practical use to histopathologists. It should be noted, however, that Sawada et $a l^{10}$ have developed a monoclonal antibody directed against a zinc binding protein (ZBPP-1) in rat Paneth cells. Paneth cell cytoplasmic granules and a population of mononuclear cells in the digestive tract mucosa stained positively with this monoclonal antibody. As these antibodies cross-react with human intestinal Paneth cells, they may become a powerful, although not readily available, tool for the study of Paneth cells in normal and disease states. Pokeweed (Phylotacca americana) lectin, which binds murine intestinal Paneth cells, is also a promising finding. ${ }^{1}$ Pokeweed lectin stained secretory granules of small intestinal Paneth cells in mice and rats, with a distribution identical with that obtained on staining with anti-lysozyme antibodies.

In the present study, the fact that CD15 and anti-lysozyme antibody both failed to highlight any Paneth cells in one case of normal colonic mucosa may be attributed to the vagaries of the fixation process.

CD15 is commonly used in histopathology laboratories, particularly for detecting ReedSternberg cells and discriminating between carcinoma and mesothelioma. ${ }^{22-25}$ CD15 (C3D-1) immunostains Paneth cells, ReedSternberg cells, Hodgkin cells, certain carcinoma cells, mature granulocytes, and dendritic reticulum cells. In common with the Leu-M1 and Tü 9 antibodies, C3D-1 reacts with an epitope involving the carbohydrate sequence 3-fucosyl-N-acetyllactosamine, also known as $\mathrm{X}$-hapten, X-determinant, or CD15 antigen. ${ }^{22-24}$

Expression of CD15 in Paneth cells poses some challenging questions about the function of this marker. The study by Lund-Johansen et $a l^{26}$ on activation of human phagocytes through carbohydrate antigens may be very helpful in this regard. According to these authors, CD15 may act as a receptor capable of signal transduction and phagocyte activation in leucocytes, as antibody cross-linking of CD15 induces moderate release of calcium ions into the cytoplasm and strong stimulation of the oxidative burst. The Paneth cell, in its proposed role as a regulator of the intestinal microbial flora, may well make use of CD15 as a receptor to trigger its antimicrobial functions.

Although less sensitive than anti-lysozyme antibody, CD15 may prove to be a valuable marker for the study of Paneth cells in the developing intestine, gastrointestinal inflammatory disease, and neoplasia of the digestive tract. Besides being present with metaplastic features in inflammatory conditions, Paneth cells with neoplastic features may also accompany adenomas and carcinomas of the stomach and intestine. ${ }^{20} 2127-29$ Thus, the Paneth cell, far from being an innocent bystander in the gut, participates in the response to a variety of inflammatory and neoplastic stimuli. Hopefully, unravelling the functional purpose of CD15 expression in Paneth cells will clarify the significance of this participation.

The authors thank Ms Maria Soria, Department of Pathology, Hospital Universitari Germans Trias i Pujol, for her technica support. Supported in part by CICYT Grant SAF 93/0441 and FIS Grant 95/1345.

1 Sandow MJ, Whitehead R. The Paneth cell. Gut 1979; 20:420-31

2 Geller SA, Thung SN. Morphologic unity of Paneth cells. Arch Pathol Lab Med 1983;107:476-9.

3 Mathan M, Hughes J, Whitehead R. The morphogenesis of the human Paneth cell. An immunocytochemical ultrastructural study. Histochemistry 1987;87:91-6.

4 Morson BC. Intestinal metaplasia of the gastric mucosa. $\mathrm{Br}$ f Cancer 1955;9:365-76.

5 Symonds DA. Paneth cell metaplasia in diseases of the colon and rectum. Arch Pathol 1974;97:343-7.

6 Nistal M, García-Rodeja E, Paniagua R. Granular transformation of Sertoli cells in testicular disorders. Hum Patho 1991;22:131-7.

7 Lee KR, Trainer TD. Adenoarcinoma of the uterine cervix of the small intestinal type containing numerous Paneth cells. Arch Pathol Lab Med 1990;114:731-3.

8 Weaver MG, Abdul-Karim FW, Srigley JR, Bostwick DG, Ro JY, Ayala AG. Paneth cell-like change of the prostate gland: A histological, immunohistochemical and electron microscopic study. Am $\mathcal{F}$ Surg Pathol 1992;16:62-8.

9 Adlakha H, Bostwick DG. Paneth cell-like change in prostatic adenocarcinoma represents neuroendocrine differentiation. Hum Pathol 1994;25:135-9.

10 Sawada M, HoriguchiY, Abujiang P, Miyake N, KitamuraY, Midorikawa O, Hiai J. Monoclonal antibodies to zinc-binding protein of rat Paneth cells. $\mathcal{F}$ Histochem Cytochem 1994;42:467-72.

11 Evans GS, Chwalinski S, Owen G, Booth C, Singh A, Potten CS. Expression of pokeweed lectin binding in murine intestinal Paneth cells. Epithelial Cell Biol 1994;3:7-15.

12 Murata K, Egami H, Kiyohara H, Oshima S, Kurizaki T, Ogawa $\mathcal{M}$. Expression of group-II phospholipase A2 in Ogawa $\mathrm{M}$. Expression of group-I phospholipase $\mathrm{A} 2$ in Mancer 1993;68: 103-11.

13 Nevalainen TJ, Gronroos JM, Kallajoki M. Expression of group II phospholipase $\mathbf{A 2}$ in the human gastrointestina tract. Lab Invest 1995;72:201-8.

14 Tan X, Hsueh W, González-Crussi F. Cellular localization of tumor necrosis factor (TNF)-alpha transcripts in normal bowel and in necrotizing enterocolitis. TNF gene expression by Paneth cells, intestinal eosinophils, and macrophages. Am f Pathol 1993;142:1858-65.

15 Schmauder-Chock EA, Chock SP, Patchen ML. Ultrastructural localization of tumour necrosis factor-alpha. Histochem $f$ 1994;26:142-51.

16 Lacasse J, Martin LH. Detection of CD1 mRNA in Paneth cells of the mouse intestine by in situ hybridization. $f$ Histochem Cytochem 1992;40:1527-34

17 Ohtsuka A, Kikuta A, Taguchi T, Murakami T. A hydrophilic resin-embedding method for light and electron microscopic detection of tissue anionic sites with cationic colloidal iron: as applied to mouse Paneth cells. Arch Histol Cytol 1993;56:423-30. 
18 Ganz T, Lehrer RI. Defensins. Curr Opin Immunol 1994;6:584-9.

19 Jones DE, Bevins CL. Defensin-6 mRNA in human Paneth cells: implications for antimicrobial peptides in host defense of the human bowel. FEBS Lett 1993;315:187-92.

20 Caruso RA, La Spada F, Casablanca G, Rigoli L. Lysozyme and mucins in gastric adenomas. F Clin Pathol 1989; 42:827-33

21 Kimura W, Futakawa N, Yamagata S, Wada Y, Kuroda A, two histological types of carcinoma of papilla of Vater. $f p n$ 7 Cancer Res 1994;85:161-6.

22 Stein H, Uchanska-Ziegler B, Gerdes J, Ziegler A, Wernet P. Hodgkin and Sternberg-Reed cells contain antigens specific to late cells of granulopoiesis. Int $\mathcal{f}$ Cancer 982;29:283-90.

23 Schienle HW, Stein H, Müller-Ruchholtz W. Neutrophil granulocytic cell antigen defined by a monoclonal antibody-its distribution within normal haemic and non-haemic tissue. F Clin Pathol 1982;35:959-66.

24 Stein H, Gerdes J, Schwab U, Lemke H, Mason DY, Ziegler
A. Identification of Hodgkin and Sternberg-Reed cells as a unique cell type derived from a newly detected small-cell population. Int $\mathcal{f}$ Cancer 1982;30:445-59.

25 Otis CN, Carter D, Cole S, Battifora H. Immunohistochemical evaluation of pleural mesothelioma and pulmonary adenocarcinoma. A bi-institutional study of 47 cases. Am ₹ Surg Pathol 1987;11:445-56.

26 Lund-Johansen F, Olweus J, Horejsi V, Skubitz KM, Thompson JS, Vilella R, et al. Activation of human phagocytes through carbohydrate antigens (CD15, sialyl-CD15, CDw17, and CDw65). F Immunol 1992;148:3221-9.

27 Gibbs NM. Incidence and significance of argentaffin and Paneth cells in some tumours of the large intestine. $\mathcal{F}$ Clin Paneth cells in some tumol $1967 ; 20: 826-31$.

28 Lev R, DeNucci TD. Neoplastic Paneth cells in the stomach-Report of two cases and review of the literature. Arch Pathol Lab Med 1989;113:129-33.

29 Odze R, Gallinger S, So K, Antonioli D. Duodenal adenomas in familial adenomatous polyposis: relation of cell differentiation and mucin histochemical features to growth pattern. Mod Pathol 1994;7:376-84. 